\title{
Respeto a los derechos de los pueblos originarios en el currículo educativo en torno a la enseñanza-aprendizaje de centros escolares de Panchimalco
}

The respect for the human rights of the indigenous peoples in the school curricula of Panchimalco

\author{
Carlos Felipe Osegueda-Osegueda \\ Miguel Angel Hernández ${ }^{2}$ \\ Georgina Sulamita Ordóñez-Valle ${ }^{3}$ \\ Noé Isaí Zamora-Mejía ${ }^{4}$ \\ Guadalupe Amparo Sigüenza-Arévalo ${ }^{5}$ \\ Universidad Tecnológica de El Salvador \\ carlos.osegueda@utec.edu.sv \\ Recibido: 21/07/17- Aceptado:10/10/17
}

\section{Resumen}

El Salvador ha sufrido rupturas en los procesos de endoculturación a lo largo de su historia, sin embargo, en los últimos años el Estado y algunos grupos sociales han posicionado en la agenda nacional el tema del reconocimiento y respeto a los derechos de los pueblos indígenas. El análisis del currículo educativo nacional y del comportamiento estudiantil en el proceso de enseñanzaaprendizaje permite comprender si en verdad existe un esfuerzo en todo el país por la revitalización de los elementos culturales y los derechos de los pueblos originarios. Se presenta un panorama desde el planteamiento teórico del papel de la educación y desde la visión de expertos culturales, directores, maestros y alumnos del tercer ciclo de educación básica de Panchimalco.

\section{Palabras clave}

Grupos étnicos - Educación; Derecho a la educación; Cambio cultural - El Salvador.

\begin{abstract}
El Salvador's endoculturation processes have undergone different breakups throughout the country's history, nonetheless, in the past recent years the state and a few socialgroups haveachieved to position theacknowledgment and respect towards the rights of the indigenous peoples within the national agenda. The analysis of the national curricula and the student behavior in the teaching-learning process allows for an understanding of whether there is a true national effort for the revitalization of those cultural elements and the rights of the Native peoples. This research introduces an overview of the situation as seen from the perspective of a theoretical approach in terms of the role of education as well as from the viewpoint of cultural experts and the junior high school principals, faculty, and students in Panchimalco.
\end{abstract}

\section{Keywords}

Ethnic groups - Education; Right to education; Cultural change - El Salvador

\footnotetext{
1 Profesor titular de la asignatura Antropología Social.

2 Estudiante instructor de la asignatura Antropología Social.

${ }^{3}$ Estudiante investigador de la Licenciatura en Antropología, Utec.

${ }^{4}$ Estudiante investigador de la Licenciatura en Antropología, Utec.

${ }^{5}$ Estudiante investigador de la Licenciatura en Antropología, Utec.
} 


\section{El papel de la educación en los cambios culturales}

La educación es esencial en el proceso culturizador. Desde la perspectiva antropológica, coopera con los cambios culturales de una sociedad. Uno de los elementos que encamina al cambio cultural es el difusionismo, corriente teórica propuesta en Norteamérica por Franz Boas, la cual se define de la forma siguiente:

Es la transmisión de elementos de una cultura a otra. Estos elementos son transmitidos por agentes que se sirven de medios identificables y están sujetos a los efectos de diferentes barreras o filtros (...) El difusionismo se refiere a cualquier hipótesis que postula el origen exógeno de la mayoría de los elementos de una cultura o subconjunto cultural específicos (Barfield, 2010).

En El Salvador, el sistema educativo es uno de los transmisores de elementos culturales extranjeros con mayor influencia. Lamentablemente, en el programa de educación, inspirado en el currículo educativo, no se percibe ningún interés por abonar al conocimiento y la difusión de la identidad cultural. En ese sentido, la endoculturación pasa a jugar un papel muy importante, convirtiéndose en la responsable de que en la sociedad existan elementos culturales similares de una generación a otra.

Marvin Harris (2004) definió endoculturación como "experiencia de aprendizaje parcialmente consciente y parcialmente inconsciente a través de la cual la generación de más edad incita, induce y obliga a la generación más joven a adoptar los modos de pensar y comportarse tradicionales".

Sin embargo, el proceso de endoculturación ha sufrido una ruptura visible en las generaciones actuales de El Salvador. Los jóvenes de hoy no replican con exactitud los elementos culturales de la generación anterior, y en muchas ocasiones los reemplazan con nuevos elementos. Este suceso es conocido como abismo generacional, según Margaret Mead (1970) expresó lo siguiente:

Hoy en día, en ninguna parte del mundo hay ancianos que sepan lo que los niños ya saben; no importa cuán remotas y sencillas sean las sociedades en las que vivan estos niños. En el pasado siempre había ancianos que sabían más que cualquier niño en razón de su experiencia de maduración en el seno de un sistema cultural.
Hoy en día no los hay. No se trata sólo de que los padres ya no sean guías, sino de que ya no existen guías, los busquemos en nuestro propio país o en el extranjero. No hay ancianos que sepan lo que saben las personas criadas en los últimos veinte años sobre el mundo en el que nacieron (Mead, citado en Harris, 2004).

\section{El currículo educativo en la formación de las nuevas generaciones}

La educación es un derecho humano fundamental, por medio del cual el individuo enriquece su conocimiento intelectual y cultural. También es considerado como un proceso exclusivo del desarrollo humano que le permite conocer y ejercitar el resto de sus derechos. Por su parte, el proceso educativo es el conjunto de actividades, planificaciones y experiencias que permiten la adquisición de contenidos culturales e intelectuales mediante la enseñanza. En la actualidad, este proceso no podría llevarse a cabo sin el sistema de planeación educativo, en el cual se definen los objetivos y las metas de una actividad educativa, con el fin de determinar los recursos y estrategias más apropiadas para su logro, todo ello se origina a partir del currículo educativo (Aguilar Morales \& Vargas-Mendoza, 2011).

En El Salvador, el currículo educativo nacional actual surge en 1994 con el nombre de "Fundamentos curriculares de la educación nacional".

En el documento se encuentra una definición de currículo, la cual expresa:

El currículo es el instrumento clave de concepción, sistematización y organización de los procesos educativos. Desarrolla y promueve acciones sociales, científicas, tecnológicas y propiamente pedagógicas, aplicadas a la práctica educativa en los diversos niveles y modalidades del sistema.

- Conlleva y expresa un conjunto de concepciones y valores respecto del ser humano, su mundo natural y social.

- Es el vehículo a través del cual se garantiza la direccionalidad e intencionalidad de una propuesta educativa que se desarrolla en un momento histórico y en un contexto social determinados (Ministerio de Educación de El Salvador [Mined], 1994). 
Una de las partes más importantes de la planeación educativa es el diseño curricular, el cual regula los contenidos de aprendizaje por nivel o grado de escolaridad y que se concibe como sigue:

El diseño curricular torna prácticos los fundamentos, principios y objetivos del currículo nacional. Es el puente entre la teoría pedagógica y la práctica en el aula. Así entendido, integra intencionalidades, contenidos, metodologías y recursos para el logro de los aprendizajes en todos los niveles y modalidades del sistema (Mined, 1994).

El diseño curricular cuenta con los siguientes instrumentos:

- planes y programas de estudio,

- recursos didácticos (textos, guías didácticas) y

- unidades de aprendizaje y módulos de formación, entre otros.

La planificación curricular se realiza por niveles, grados, ciclos, unidades, módulos de aprendizaje, etc. Se perfecciona constantemente con la práctica de todos los actores de la comunidad educativa. "Los Fundamentos curriculares de la educación nacional hacen explícitos los objetivos, los principios que la orientan, las fuentes que la nutren y los aspectos técnicos considerados en el diseño curricular." (Mined, 1994.)

\section{Enfoque intercultural en la educación}

En América Latina ha existido una intensa lucha por la incorporación de una formación escolar que incluya los valores indígenas. Según López y Sichra (2004), los movimientos indigenistas han incidido en países que tienen una población indígena mayoritaria, como Bolivia, que es del $65 \%$, pero también en aquellos de población indígena muy minoritaria, como en Brasil, que representa el 0,3\%. Incluso en países como Costa Rica, Chile y Argentina, que se habían considerado por mucho tiempo totalmente castellanizados, han tenido que ceder a las manifestaciones y a las luchas de sus poblaciones indígenas (López, 2001).

En El Salvador, tanto en el currículo educativo como en el diseño curricular no se contemplan a los pueblos originarios salvadoreños entre sus objetivos y contenidos. Si bien en la materia de Estudios Sociales y Cívica se presentan temas relacionados con la cultura, el patrimonio y los pueblos originarios, no existe una profundización en cuanto a la historia, su origen y cultura de los pueblos indígenas que habitan El Salvador, evidenciando una falta de interés por parte del Mined en promover y preservar la identidad cultural de la nación. Según el Consejo Nacional para la Cultura y el Arte (2003), actualmente Secretaría de Cultura de la Presidencia, "al marginar los elementos de la cultura autóctona, refuerzan la creencia de que la 'superación' sólo se logra a través del aprendizaje de culturas ajenas".

A diferencia de El Salvador, en el currículo educativo de Guatemala, en sus objetivos, antepone el elemento multicultural. El primer objetivo dice textualmente:

Reflejar y responder a las características, necesidades y aspiraciones de un país multicultural, multilingüe y multiétnico, respetando, fortaleciendo y enriqueciendo la identidad personal y la de sus pueblos como sustento de la unidad en la diversidad (Ministerio de Educación de Guatemala, 2005).

A la comunidad también se le asigna una misión decisiva en el Currículo Nacional Base de Guatemala. Se denota una conexión muy fuerte entre comunidad y escuela, una inseparable de la otra. Esta dinámica propicia un exitoso proceso de endoculturación. Es decir, las generaciones de mayor edad pueden transmitir a las nuevas generaciones, en un ambiente favorable, los elementos de su cultura. El currículo dice lo siguiente sobre la comunidad:

Participa activamente en el fortalecimiento del proceso educativo propiciando la relación de la comunidad con el Centro Educativo: su idioma, su cultura, sus necesidades y sus costumbres. En otras palabras, promueven el acercamiento de la escuela a la vida (Ministerio de Educación de Guatemala, 2005).

Todo el entorno en que se desarrolla el estudiante tiene un valor específico para la educación. Podría decirse que el Currículo Nacional de Guatemala reafirma en cada actor y área de la educación valores como el pluralismo, la equidad y la pertinencia.

El Estado Plurinacional de Bolivia es otro digno ejemplo de la incorporación del respeto y reconocimiento de los 
derechos de los pueblos indígenas en el currículo educativo. En su Currículo Base del Sistema Educativo Plurinacional, el Ministerio de Educación presenta, como un eje transversal en todo el documento, el tema de los pueblos originarios. En el segmento titulado "Caracterización general de la educación en Bolivia" comienza diciendo que "la educación desarrollada en los pueblos indígenas originarios, cuya presencia es anterior a la Colonia, fue diversa". Es decir, anuncia que los fundamentos de la educación deben buscarse en la misma diversidad de los pueblos que antecedieron a la Conquista. En su apartado "Bases del currículo" expresa lo siguiente:

El currículo tiene como una de sus fuentes y bases al conocimiento, la sabiduría, saberes y valores de los pueblos y naciones indígenas originarios, desarrollados a partir de una educación comunitaria. Esta forma de enseñanza y aprendizaje se vivió como una experiencia y no tanto como una metodología sistematizada, pues estaba vinculada directamente con la vida de las comunidades (Ministerio de Educación de Bolivia, 2012).

Países como Guatemala y Bolivia demuestran avances significativos en un tema que estuvo olvidado por muchos años. Se presentan como guías a los países que avanzan a pasos lentos hacia la aprobación de políticas públicas y marcos legales para los derechos de los pueblos originarios. En materia educativa, también superan a los demás países de la región y marcan una pauta para los países que pretenden impulsar la construcción y el fortalecimiento de la identidad cultural a través de la educación.

\section{Método y proceso de investigación}

Para la elaboración de la investigación se implementó el método cualitativo desde el enfoque etnográfico. Haciendo uso de la técnica de la entrevista semiestructurada fue posible conocer la percepción de los docentes y estudiantes de dos centros escolares del municipio de Panchimalco, en torno al respeto hacia los derechos de los pueblos originarios.

El método etnográfico permite la descripción de un grupo social a partir del trabajo de campo, y lo recopilado en este debe organizarse, describirse, analizarse e interpretarse para construir explicaciones acerca de un tema en particular (Kottak, 2011).
El proceso de investigación constó de tres etapas: 1) Consulta de fondos documentales, que hizo posible la construcción tanto del marco teórico-conceptual como del históricoreferencial, a partir de los cuales se expusieron conceptos básicos, teorías vigentes en torno a la educación, posturas y antecedentes históricos del municipio de Panchimalco y pueblos indígenas, entre otros. 2) Establecimiento del contacto con diversos informantes e instituciones que proporcionaron respuestas a los principales interrogantes de la investigación. Con el fin de conocer en mayor profundidad el Estado del derecho y el respeto hacia los pueblos originarios en el currículo nacional educativo, se visitaron dos instituciones: Centro Escolar "Cantón El Cedro" y el Complejo Educativo "Goldtree Liebes". Los criterios que debieron cumplir los informantes incluyeron ser docente de la asignatura de Estudios Sociales y Cívica de noveno grado de educación básica y tener más de un año laborando en el centro escolar; en el caso de los participantes que conformaron la población estudiantil, tener entre 13 y 17 años, que cursaran séptimo, octavo y noveno grado, siendo la población de interés estos últimos. 3) Sistematización y socialización de los resultados obtenidos. La información recolectada de las entrevistas a docentes y a estudiantes se analizó y comparó con el fin de distinguir las similitudes y diferencias en las percepciones individuales orientadas hacia el derecho y respeto de los pueblos originarios, esto dio lugar a la relación con los marcos teórico-conceptual e histórico-referencial, con lo cual fue posible dar respuestas a nuestros interrogantes partiendo de la situación vivida por los participantes de la investigación.

Para la elaboración de los instrumentos de investigación se realizó una matriz de congruencia a partir de la cual fue posible obtener las preguntas necesarias para la guía de entrevista y el grupo focal.

Como se mencionó anteriormente, las técnicas del método etnográfico que se utilizaron fueron entrevistas semiestructuradas y grupos focales. Con ambas técnicas se pretendió conocer de primera mano y en profundidad la situación actual del respeto y derecho hacia los pueblos originarios, conociendo así diversos puntos de vista. 


\section{Resultados}

1. Contenidos del diseño curricular que abonan al respeto de los derechos de los pueblos originarios

Uno de los ocho objetivos que contiene el currículo educativo nacional para la asignatura de Estudios Sociales es "conseguir que se identifiquen las raíces históricas y la riqueza cultural para favorecer el desarrollo de la identidad y autoestima personal, comunitaria, nacional, centroamericana y latinoamericana" (Mined, 1994)". Sin embargo, este propósito escasamente se ve reflejado en los contenidos del programa de estudio y en los libros de texto. Mientras otros países latinoamericanos desarrollan una educación intercultural, en El Salvador apenas se pretende rescatar elementos de las raíces culturales.

El profesor de Estudios Sociales del Centro Escolar "El Cedro", ubicado en la zona rural, Noel Anzora, aclara que el programa de Estudios Sociales y Cívica propuesto por el diseño curricular para tercer ciclo solo abarca algunos contenidos relacionados con identidad cultural, cultura y etnia:

Directamente no, sí en el de octavo, pero en el de noveno grado no está, yo retomo siempre el tema en noveno grado, pero no, no está contemplado directamente. Habría que revisarlo, pero no, yo creo que no está. Lo estuve revisando y no encontré nada. Específicamente el respeto a los derechos de los pueblos indígenas está en el programa de octavo, pero en el de noveno no, no está (Anzora, 2017).

Rita de Araujo (2017), jefa del Departamento de Pueblos Originarios de la Secretaría de Cultura de la Presidencia, considera que el motivo por el cual el país no incorporó la temática indígena con su debida importancia en el currículo educativo es porque históricamente ha existido un interés generalizado en la población por considerarse mestiza. Explica que antes, en el país, se "evidenciaba más a lo criollo, a todo lo que fue surgiendo a partir de la invasión de los españoles (...) Los negros fueron todavía mejor tratados que los indígenas" (ibídem, 2017).

En el programa de Estudios Sociales de noveno grado, los derechos humanos y la cultura son abordados de manera general. Esta temática aparece por primera vez en tercer ciclo en el programa de Estudios Sociales de séptimo grado, donde se brindan los conceptos generales acerca del tema, sin darles especial importancia a los pueblos originarios salvadoreños. En los libros de texto propuestos por el diseño curricular para noveno grado - de la Colección Cipotas y Cipotes-, únicamente la unidad tres aborda una temática cultural.

El profesor Anzora admite que, si bien la importancia que se le da a la identidad cultural en el programa de Estudios Sociales es muy poca; el maestro puede profundizar más en los temas relacionados a las raíces culturales.

Dentro del programa de estudio, la importancia creo que es bien poca, a lo sumo, un par de contenidos ahí, que hablan sobre ello, y si revisáramos el libro de la Colección Cipotes, que es el libro que da el Ministerio de Educación para nosotros, es bien escueto, prácticamente no aborda nada sobre el tema, entonces queda a título personal abonar un poco más a eso (Anzora, 2017).

\section{Estrategias metodológicas en torno a la enseñanza y fomento al respeto de los pueblos originarios}

Las palabras del maestro Anzora dan pie al otro elemento sustancial en este tema: el proceso de enseñanzaaprendizaje. La labor del profesor y la metodología que este implemente se vuelve vital en la transmisión de conocimientos acerca de la historia de las raíces culturales salvadoreñas. Hilda de Álvarez (2017), jefa del Departamento de Investigación e Innovación Educativa del Mined, considera que no puede ser únicamente el docente de Estudios Sociales quien aborde la temática de la identidad cultural, sino que esta debe estar presente en todas las asignaturas:

Yo creo que en primer lugar el docente, y no solamente el de Estudios Sociales, porque esa es una de las cosas que nosotros queremos ver. A veces cuando se habla de identidad y de los temas de identidad cultural o de cultura de manera general se cree que solamente en Estudios Sociales se tendrían que ver estas temáticas, ¿verdad?; y no, la identidad cultural nosotros la podemos ver en las diferentes asignaturas (Álvarez, 2017).

El proceso de enseñanza-aprendizaje es una responsabilidad que recae principalmente en los docentes 
y directores de los centros educativos. La directora del Centro Escolar "Goldtree Liebes", Sayda Vargas, se considera a sí misma comprometida con la recuperación de las tradiciones culturales, a fin de generar identidad cultural en sus estudiantes. El planteamiento de Vargas incluye un elemento importante en la dinámica, en el que se asume un compromiso a nivel de centro más que en una materia específica: "Mi papel es que no se pierda. Bueno, no que no se pierda, recuperarlo, porque de hecho lo desconocen; que los chicos recuperen el conocimiento de dónde venimos, quiénes somos y por qué estamos aquí y para qué" (Vargas, 2017).

En el municipio de Panchimalco se advierte un marcado interés en la dinámica de los centros escolares por la enseñanza de las raíces culturales. El profesor Noel Anzora afirma que sus métodos de enseñanza para acercar a los alumnos conocimientos sobre temas indígenas son muy variados: "Utilizo mucho videos, previo a una clase o al final del contenido. Este tipo de clase, a mí me gusta hacerlas bastante positivas" (Anzora, 2017).

El profesor Anzora explica que los estudiantes algunas veces se muestran sorprendidos cuando se les enseña sobre la diversidad cultural. Por ese motivo, él recurre a metodologías que despierten el interés en los jóvenes; y lo expresa de la siguiente manera:

Me gusta mucho hablarles sobre identidad, el derecho a los pueblos indígenas, la importancia de la tolerancia hacia estos pueblos, sentirse identificados. Pero más que todo, mi estrategia en este tema es la clase positiva, ponerles ejemplos, como le mencioné los videos, incluso les pongo fotografías no solo de El Salvador, sino que también de América y les pido que me digan, por ejemplo, de alguna tribu brasileña, que salen fotos donde están desnudos, y pregunto ¿qué me dicen de estas fotos? ¡Huy no, profe, están chulones!, responden. Entonces, y ahí voy armando mi discurso sobre este tema (Anzora, 2017).

3. Respeto y derechos hacia los pueblos originarios en las actividades estudiantiles del tercer ciclo del municipio de Panchimalco

Los estudiantes del nivel de tercer ciclo de educación básica del municipio de Panchimalco muestran cómo son consideradas las personas indígenas en la cotidianidad. Reconocen que el término indígena ha tomado connotaciones peyorativas. El indígena suele asociarse con lo ridículo: "Hay veces, cuando uno hace una bayuncada, no sé; o algo como que quieren dar a entender lo que hacían antes las personas... sin madurez o algo así" (Estudiantes del C. E. "Goldtree Liebes", 2017).

En cuanto a la definición del indígena, los estudiantes de la zona urbana lo asocian con el pasado, al lenguaje y a la vestimenta. En algunos casos la imagen del indígena tiende a verse de manera un tanto negativa: "Ser indígena como que es, como que los tiempos de antes, eh, que se vestían, el tipo de comida, o sea, era muy diferente, cocinaban así, en el suelo, andaban vestidos con ropas así, casi que desnudos" (Estudiantes del C. E. "Goldtree Liebes", 2017).

Para el antropólogo Guillermo Cuéllar (2017), plantear la respuesta a lo que significa ser indígena en El Salvador supone una misión intrincada:

Reconocer que hay un amplio mestizaje, y que dentro de este podemos descubrir, pero muy profundamente, algunos rasgos que todavía nos quedan y que no han sido enfocados tan específicamente por estudiosos, por ejemplo, la jerarquización de la familia (Cuéllar, 2017).

Las prácticas cotidianas estudiantiles inciden en el fomento del respeto hacia los derechos de los pueblos originarios, lamentablemente en el día a día de los estudiantes, el indígena sigue siendo estigmatizado. La relación entre el término indígena o indio se asocia con el menosprecio. Esta una manifestación muy común entre los salvadoreños, según lo expresa Hugo de Burgos:

Está marcada propensión a acentuar, exagerar y hasta inventar vínculos indígenas cuando se trata de humillar, menospreciar y desvirtuar a una persona o grupo. (...) Frases y conceptos claramente racistas simultáneamente sostienen y expresan el desprecio con el que muchas personas salvadoreñas típicamente se refieren a su propia herencia fenotípica y cultural (Burgos, 2010).

Pese a los estereotipos existentes en la población estudiantil de El Cedro (Los Planes, por lindar con Panchimalco), los mismos estudiantes reconocen que en 
la materia de Estudios Sociales están aprendiendo mucho sobre el tema indígena. A nivel de centro escolar se pueden encontrar interesantes iniciativas, por parte de maestros y directores, para llevar a cabo actividades extracurriculares que fomentan el conocimiento y el respeto hacia los pueblos originarios. Sayda Vargas manifiesta la importancia que le han dado al rescate de las raíces culturales a través de diversas iniciativas.

Tuvimos aquí un festival gastronómico el año pasado y trajimos todo el origen de nuestra comida típica, cómo fue, de dónde nació e invitamos a personas que son, este, cómo se les dice, que ellos se llaman ser indígenas. Trajimos unos invitados, y los señores explicaron hasta cómo hacían sus instrumentos, una exposición de instrumentos antiguos, de cómo los elaboraban; y él les explicaba el significado y los sonidos de cada cosa, la moneda, por qué se llama, por qué hay escudos y banderas, por qué Panchimalco se llama "lugar de escudos y banderas" (Vargas, 2017).

\section{Conclusiones}

Los problemas de invisibilidad y vulneración hacia los derechos de los indígenas en El Salvador no son recientes ni aislados, pero han empeorado como consecuencia directa de un proceso excluyente en lo económico, social y cultural desde la Conquista hasta la actualidad. Una de las claras causas de este mal proceso es el Estado y Gobierno; es la poca voluntad hacia la divulgación de los derechos indígenas, por lo que es de suma importancia crear un verdadero plan de Nación que incluya una educación intercultural para la población del país. A partir de esta investigación, se pretendió evidenciar lo que sucede en las aulas de clases; y no solamente en el municipio de Panchimalco, sino más bien a nivel de todos los centros educativos del país, ya que el currículo y el diseño curricular tienen muchos vacíos con respecto a este tema.

\section{Referencias}

Aguilar Morales, J.E. \& Vargas-Mendoza, J.E. (2011). "Planeación educativa y diseño curricular: un ejercicio de sistematización". En Boletín Electrónico de Investigación de la Asociación Oaxaqueña de Psicología A.C., pp. 53-64. Recuperado de www. conductitlan.net/notas_boletin_investigacion/140_ planeacion_educativa_curriculum.pdf
Álvarez, H. (04 de abril del 2017). “Procesos de formación docente para la preservación del náhuat" (N. Zamora \& G. Ordóñez, entrevistadores).

Anzora, N. (31 de marzo del 2017). “Procesos de enseñanzaaprendizaje en la materia de estudios sociales hacia los pueblos originarios" (N. Zamora, A. Sigüenza \& G. Ordóñez, entrevistadores).

Barfield, T. (2010). Diccionario de Antropología. Madrid, España: Siglo XXI de España editores.

Consejo Nacional para la Cultura y el Arte (2002). "Perfil de los pueblos indígenas en El Salvador". En El Banco Mundial. Recuperado de http://documents. worldbank.org/curated/en/939901468234885618/pdf /647480PUBOPortugese00PUBLIC0.pdf

Cuéllar, G. (30 de marzo de 2017). "Identidad cultural en El Salvador" (N. Zamora, A. Sigüenza \& G. Ordoñez, entrevistadores)

De Araujo, R. (30 de marzo del 2017). "Respeto y derecho hacia los pueblos originarios de El Salvador" (N. Zamora, A. Sigüenza \& G. Ordóñez, entrevistadores).

De Burgos, H. (2010). "Racismo, símbolos de la belleza, autoestima y salud mental en El Salvador". En Identidades. Revista de Ciencias Sociales y Humanidades. 1, (1), pp. 8-31.

Grupo focal con estudiantes del Centro Escolar "El Cedro" (31 de marzo del 2017). "Percepción estudiantil hacia los pueblos originarios" (N. Zamora, A. Sigüenza \& G. Ordóñez, entrevistadores).

Grupo focal con estudiantes del Centro Escolar "Goldtree Liebes" (31 de marzo del 2017). "Percepción estudiantil hacia los pueblos originarios" (N. Zamora, A. Sigüenza \& G. Ordoñez, entrevistadores).

Harris, M. (2004). Antropología cultural. Madrid, España: Alianza Editorial.

Kottak, C. (2011) Antropología Cultural. México, D.F: McGraw-hill. López, L.E. (2001). "La cuestión de la interculturalidad y la educación latinoamericana". Unesco.

López, L. y Sichra, I. (2004). “La educación en áreas indígenas de América Latina: balances y perspectivas". En Educación en la diversidad. Experiencias y desafíos en la Educación Intercultural. Buenos Aires: IIPE

Ministerio de Educación de Bolivia (2012). Currículo Base del Sistema Educativo Plurinacional.

Ministerio de Educación de El Salvador (1994). "Fundamentos curriculares de la educación nacional". San Salvador, El Salvador.

Ministerio de Educación de Guatemala (2005). Currículo Nacional Base.

Vargas, S. (31 de marzo del 2017). "Iniciativa en los centros escolares urbanos en la conservación de la identidad cultural" (N. Zamora, A. Sigüenza \& G. Ordóñez, entrevistadores). 